Ann. Biol. anim. Bioch. Biophys., 1972, 12 (r), 69-76.

\title{
FACTEURS ALIMENTAIRES FAVORABLES A LA STÉATOSE PAR SURALIMENTATION DU POULET
}

\author{
I. HASSAN et B. LECLERCQ
}

Station de Recherches avicoles, Centre de Recherches de Tours, I. N. R. A., 37 - Nouzilly

\section{RÉSUMÉ}

Les effets de divers composants du régime ont été étudiés chez des poulets suralimentés grâce à une machine à gaver. Plus le taux protidique est élevé, plus les foies sont lourds et riches en protéines et en lipides. L'incorporation d'huile d'arachide à l'aliment permet d'augmenter encore l'ingestion de nutriments énergétiques; elle aggrave l'état de stéatose sans modifier le poids du foie. Ni l'amélioration de la valeur biologique par la lysine, ni l'addition de choline ne peuvent réduire l'hépatomégalie et la stéatose. En fin, la suralimentation par un régime complet n'a pas d'effets significativement différents de ceux du maîs. La stéatose de l'oiseau suralimenté ne parâ̂t donc pas due à une déficience du régime en protéines ou en facteurs lipotropes mais à une synthèse accrue de lipides. Un apport correct de protéines semble nécessaire pour permettre l'activité anabolique intense de l'organe et le maintien de l'intégrité cellulaire.

\section{IN'TRODUC'TION}

La suralimentation par gavage des oiseaux provoque des changements profonds dans la composition du foie. Elle entraîne, au début, un accroissement important des constituants actifs (protéines, acides nucléiques, phospholipides) associée à une lipogenèse intense ; par la suite, seuls les triglycérides continuent de s'accumuler ( $L_{\text {ECLEERCQ }}$ et al., I968). Ce résultat obtenu chez l'Oie a conduit BLUM et al., (I97I) a envisager l'élévation du taux protidique de l'aliment de gavage pour favoriser la synthèse hépatique de protéines et l'anabolisme qui l'accompagne et accroître ainsi le poids des foies. Grâce à cette étude, il a été clairement démontré que l'élévation du taux protidique favorise la stéatose.

Nous présentons ici les premiers résultats obtenus chez le Poulet. Cette espèce a été retenue parce qu'elle constitue un matériel expérimental commode. Une étude nutritionnelle et biochimique a pu ainsi être réalisée plus aisément que chez 1'Oie. 


\section{MATÉRIEL ET MÉTHODES}

Tous les animaux, mâles ou femelles, sont issus de croisements commerciaux destinés à la production de poulets de chair. Ils reçoivent à volonté des aliments complets et équilibrés jusqu'au début du gavage. Ils sont alors répartis en lots homogènes pour le poids vif. Le gavage est réalisé deux fois par jour grâce à une machine fabriquée spécialement dans ce but et qui permet de faire ingérer l'aliment sous forme de bouillie contenant $50 \mathrm{p}$. Ioo de matières sèches. Dans la mesure où ils le supportent, tous les animaux ingèrent la même quantité d'aliment, représentant de $r, 5$ à 2 fois la consommation journalière normale du poulet de chair à l'âge considéré. La quantité exactement ingérée à chaque repas est déterminée par pesée de l'animal avant et après gavage. Les quantités totales d'aliment consommé seront indiquées pour chaque expérience. De l'eau est mise en permanence à la disposition des sujets. La composition des régimes fait l'objet du tableau $\mathrm{I}$.

\section{TABLEAU I}

Composition des régimes (en p. roo)

\begin{tabular}{|c|c|c|c|c|c|c|c|}
\hline & $T$ & TPH & TPB & TEH & VB & VBC & C \\
\hline 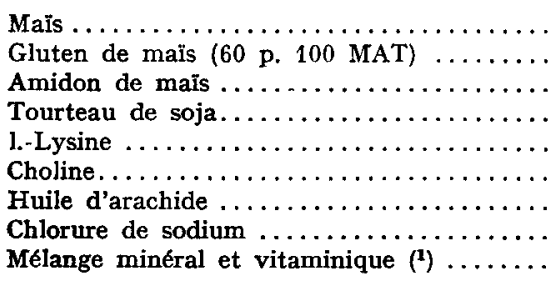 & 99,5 & $\begin{array}{l}89,5 \\
10\end{array}$ & $\begin{array}{l}79,5 \\
20\end{array}$ & $\begin{array}{l}10 \\
0,5\end{array}$ & $\begin{array}{l}89,1 \\
10\end{array}$ & $\begin{array}{l}0,4 \\
0,2 \\
0,5\end{array}$ & $\begin{array}{c}67,0 \\
15,0 \\
10,0 \\
0,15 \\
5,6 \\
0,4 \\
2,0\end{array}$ \\
\hline $\begin{array}{l}\text { Taux protidique }\left({ }^{2}\right) \ldots \ldots \ldots \ldots \ldots \ldots \ldots \ldots \ldots \\
\text { Teneur en lysine }\left({ }^{8}\right) \ldots \ldots \ldots \ldots \ldots \ldots \ldots \ldots \\
\text { Teneur en energie métabolisable }(\mathrm{cal} / \mathrm{kg}) \ldots \ldots\end{array}$ & $\begin{array}{r}9,5 \\
0,27 \\
\mathbf{3 3 5 0}\end{array}$ & $\begin{array}{r}14,2 \\
\mathbf{0 , 3 9} \\
\mathbf{3} \mathbf{3 4 0}\end{array}$ & $\begin{array}{r}7,8 \\
0,22 \\
3420\end{array}$ & $\begin{array}{r}8,7 \\
0,25 \\
\mathbf{3 8 7 0}\end{array}$ & $\begin{array}{r}14,5 \\
0,78 \\
3340\end{array}$ & $\begin{array}{r}14,5 \\
0,78 \\
3340\end{array}$ & $\begin{array}{r}19,9 \\
0,73 \\
3 \mathbf{4 3 0}\end{array}$ \\
\hline
\end{tabular}

( $\left.{ }^{1}\right)$ Composition du mélange minéral et vitaminique (pour $100 \mathrm{~kg}$ aliment) : phosphate bicalcique $800 \mathrm{~g}$; carbonate de calcium : $1000 \mathrm{~g}$; vitamine $\mathrm{A}: 375000 \mathrm{UI}$; vitamine $\mathrm{D}_{3}: 50000 \mathrm{UI}$; vitamine $\mathrm{K}: 0,5 \mathrm{~g}$; riboflavine : $1 \mathrm{~g}$; cyanocobalamine : 10 ; vitamine $\mathrm{E}: 1,2 \mathrm{~g}$.

(i) Mesuré.

(8) Calcule d'après Pion R. et Fauconneau G., A. E. C. Cahier no 6 (1966).

\section{Expérience no 1}

Les animaux sont âgés de 5 semaines. La durée du gavage est de 7 jours. Chaque lot comprend 5 coquelets et 5 poulettes. On utilise trois régimes déterminant les trois lots : $T$ constitué de maîs seul, TPH renfermant du maïs et Io p. Ioo de gluten de maïs et TPB à base de maĩs et d'amidon de mais (20 p. IOo). Certes, la composition en acides aminés du gluten n'est pas exactement la même que celle des protéines totales du maïs. Cependant, on peut admettre qu'il est possible d'étudier ainsi l'effet du taux protidique sans changement notable de la valeur biologique des protéines.

\section{Expérience no 2}

Le protocole de cette expérience est identique au précédent si ce n'est que les animaux sont gavés I I jours et âgés de ro semaines. 


\section{Expérience $n^{0} 3$}

L'objectif est le même que celui des deux premières expériences. Toutefois, il ne s'agit que de poulettes ; les essais précédents ayant montré que les réponses des coquelets étaient très hétérogènes. De plus, on n'a retenu que les lots extrêmes : TPH et TPB. Le gavage dure Ir jours sur des animaux âgés de ro semaines à raison de ro sujets par lot.

\section{Expérience $n^{\circ} 4$}

Deux lots de Io poulettes de ro semaines sont gavés pendant quinze jours avec soit le régime $T$ (maïs seul), soit le régime TEH (maïs + huile). Les animaux ingérant sensiblement la même quantité d'aliment, ceux du lot TEH absorbent plus de nutriments énergétiques que ceux du lot $\mathbf{T}$.

\section{Expérience $n^{\circ} 5$}

Cette expérience a pour but de déterminer l'effet de la valeur biologique des protéines alimentaires et celui de la choline sur le poids et la composition du foie. Les régimes utilisés sont : TPH à base de maïs et de gluten, qui présente un taux protidique élevé mais une mauvaise valeur biologique ; VB identique au précédent si cen'est qu'il est supplémenté en lysine, facteur limitant primaire ; VBC identique au lot VB mais supplémenté en choline. Le gavage dure I 5 jours. Chaque lot est constitué de 6 poulettes âgées de ıo semaines.

\section{Expérience $n^{0} 6$}

Elle comporte deux lots de 8 poulettes âgées de Io semaines. Les animaux sont gavés I 5 jours avec l'un des deux régimes suivants : T régime témoin composé exclusivement de maîs et $\mathrm{C}$ régime complet supplémenté en protéines (gluten et soja), minéraux et vitamines.

Les animaux sont sacrifiés 16 heures après le dernier gavage par section des veines jugulaires. Le foie est immédiatement prélevé et pesé. Il est ensuite lyophilisé, ce qui permet de déterminer la matière sèche. Le dosage des protéines est effectué par la méthode de Kjeldahl et celui des lipides par la méthode de Folch.

\section{RÉSULTATS}

La stéatose et 1'hépatomégalie observées dans ces expériences sont moins prononcées que celles décrites chez l'Oie. L'hétérogénéité des réponses au gavage est aussi importante chez les deux espèces. Ces deux inconvénients limitent parfois la précision des observations mais n'empêchent cependant pas de tirer des conclusions significatives.

\section{Expérience $n^{\circ} 1$}

Les résultats de la première expérience figurent dans le tableau 2. On peut constater que le poids du foie est d'autant plus important que le taux protidique est élevé. La différence n'est cependant significative au seuil o,05 qu'entre le lot TPH (mais + gluten) et les deux autres lots T (mais) et TPB (mais + amidon). Les effets sont les mêmes sur la matière sèche du foie, les lipides totaux et les protéines totales, bien que les seuils de signification soient quelque peu différents. Les animaux étant jeunes, leur croissance relative conserve, dans cet essai, une valeur élevée. Les régimes n'ont pas, de ce point de vue, le même effet. Le gain de poids vif pendant la période de gavage est d'autant plus prononcé que le taux protidique est élevé. On peut penser alors que les différences de poids des foies s'expliquent par les différences de poids 
vif en fin de gavage. Les résultats de l'analyse de covariance rapportés dans le tableau 3 montrent qu'il n'en est rien. Il en ressort également une interaction significative entre l'effet " sexe " et l'effet " régime " qui s'explique par le fait que les poulettes réagissent beaucoup plus à l'élévation du taux protidique que les coquelets.

\section{TABLEAU 2}

Influence du taux protidique de l'aliment sur le poids et la composition du foie et sur la croissance des poulets ( $\delta$ et $q$ en nombre égal) à l'âge de 5 semaines

(Expérience no $\mathrm{I}: 7$ jours de gavage)

\begin{tabular}{|c|c|c|c|c|c|c|c|c|}
\hline Lot & $\begin{array}{l}\text { Poids } \\
\text { du foie } \\
\text { (g) }\end{array}$ & $\begin{array}{c}\text { Matières } \\
\text { sèches } \\
\text { du foie } \\
\text { (g) }\end{array}$ & $\begin{array}{l}\text { Lipides } \\
\text { totaux } \\
\text { du foie } \\
\text { (g) }\end{array}$ & \begin{tabular}{|} 
Protéines \\
totales \\
du foie \\
(g)
\end{tabular} & $\begin{array}{c}\text { Poids vif } \\
\text { final } \\
\text { (g) }\end{array}$ & $\begin{array}{l}\text { Gain de } \\
\text { poids } \\
\text { (g) }\end{array}$ & $\begin{array}{c}\text { Énergie } \\
\text { métaboli- } \\
\text { sable } \\
\text { ingérée } \\
\text { (calories) }\end{array}$ & $\begin{array}{l}\text { Protéines } \\
\text { ingérées } \\
\text { (g) }\end{array}$ \\
\hline $\begin{array}{l}T \text { (maïs) } \ldots \ldots \ldots \ldots \\
\text { TPH (mais }+ \text { gluten) } \ldots \\
\text { TPB (maïs }+ \text { amidon) } \ldots\end{array}$ & $\begin{array}{l}37,1^{b} \\
42,7^{a} \\
33,2^{b}\end{array}$ & $\begin{array}{l}11,9^{b} \\
14,6^{a} \\
10,6^{b}\end{array}$ & 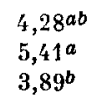 & $\begin{array}{l}6,07^{b} \\
6,70^{a} \\
5,51^{c}\end{array}$ & $\begin{array}{r}978 \\
100^{\prime} \\
908\end{array}$ & $\begin{array}{l}+174^{a} \\
+192^{a} \\
+127^{b}\end{array}$ & $\begin{array}{ll}2 & 150 \\
2 & 140 \\
2 & 340\end{array}$ & $\begin{array}{l}6,06 \\
9,10 \\
5,34\end{array}$ \\
\hline
\end{tabular}

Les moyennes accompagnées d'une même lettre ne sont pas significativement différentes au seuil de 0,05 .

\section{TABLEAU 3}

Analyse de la covariance du poids du foie en fonction du poids vif final des animaux

(Expérience no $\mathrm{I}$ )

\begin{tabular}{|c|c|c|c|c|c|}
\hline Source de variation & d. 1 . & $\begin{array}{c}\text { Somme des carrés } \\
\text { du poids vif }\end{array}$ & $\begin{array}{l}\text { Somme des carrés } \\
\text { du poids du foie }\end{array}$ & $\begin{array}{l}\text { Somme } \\
\text { des } \\
\text { produits }\end{array}$ & $\begin{array}{l}\text { Carrés moyens } \\
\text { du poids du foie } \\
\text { réduit }\end{array}$ \\
\hline $\begin{array}{l}\text { Régime } \ldots \ldots \ldots \ldots \ldots \ldots \\
\text { Sexe } \ldots \ldots \ldots \ldots \ldots \ldots \\
\text { Interaction régime } \times \text { sexe } \\
\text { Erreur } \ldots \ldots \ldots \ldots \ldots \ldots\end{array}$ & $\begin{array}{r}2 \\
1 \\
2 \\
24\end{array}$ & $\begin{array}{rl}48 & 902 \\
56 & 334 \\
3 & 231 \\
77 & 430\end{array}$ & $\begin{array}{r}484,2 \\
1,8 \\
208,8 \\
575,2\end{array}$ & $\begin{array}{r}4550,2 \\
-116,2 \\
-368,7 \\
2419,6\end{array}$ & $\begin{array}{c}87,6^{*} \\
37,7 \\
116,1^{*} \\
23,8\end{array}$ \\
\hline
\end{tabular}

* Effet significatif au seuil $5 \%$

\section{Expérience no 2}

On observe au cours de cette expérience des résultats, présentés dans le tableau 4 , qui sont semblables à ceux de l'essai précédent. Toutefois, dans cet essai les différences ne sont pas significatives entre lots gavés. L'hétérogénéité des résultats enregistrés 
chez les mâles en est partiellement la cause. Par ailleurs, la présence d'animaux non gavés permet d'apprécier l'effet du gavage : augmentation des lipides totaux mais aussi des protéines totales.

\section{TABLEAU 4}

Influence du taux protidique de l'aliment sur le poids et la composition du foie chez des poulets ( $\delta$ et ${ }^{\star}$ en nombre égal) âgés de 10 semaines

(Expérience $n^{\circ} 2$ : 1 I jours de gavage)

\begin{tabular}{|c|c|c|c|c|c|c|c|}
\hline Lot & $\begin{array}{c}\text { Poids } \\
\text { du foie } \\
\text { (g) }\end{array}$ & $\begin{array}{c}\text { Matières } \\
\text { sèches } \\
\text { du foie } \\
\text { (g) }\end{array}$ & $\begin{array}{c}\text { Protéines } \\
\text { totales } \\
\text { (g) }\end{array}$ & $\begin{array}{c}\text { Lipides } \\
\text { totaux } \\
\text { (g) }\end{array}$ & $\begin{array}{c}\text { Poids vif } \\
\text { final } \\
\text { (g) }\end{array}$ & $\begin{array}{c}\text { Gain } \\
\text { de poids } \\
\text { (g) }\end{array}$ & $\begin{array}{c}\begin{array}{c}\text { Aliment } \\
\text { ingéré }\end{array} \\
\text { (g) }\end{array}$ \\
\hline $\mathrm{T}$ (mais) & $59,1^{b}$ & $27,0^{b}$ & $16,30^{a}$ & $8,27^{b}$ & 2321 & $+282^{b}$ & 1916 \\
\hline TPH (mais + gluten) & $61,5^{b}$ & $29,0^{b}$ & $17,35^{a}$ & $9,93^{b}$ & 2442 & $\begin{array}{r}373^{a} \\
+\end{array}$ & 1907 \\
\hline TPB (mais + amidon) & $58,0^{b}$ & $25,5^{a b}$ & $16,35^{a}$ & $6,10^{a b}$ & 2320 & $+278^{b}$ & 1933 \\
\hline Non gavés $\ldots \ldots \ldots \ldots$ & $44,7^{a}$ & $21,8^{a}$ & $15,85^{a}$ & $4,23^{a}$ & 2297 & & \\
\hline
\end{tabular}

Les moyennes accompagnées de la même lettre ne sont pas significativement différentes au seuil 0,05.

\section{Expérience $n^{\circ} 3$}

Les résultats de cette expérience font 1'objet du tableau 5 . Il existe cette fois un effet très prononcé et significatif du taux protidique sur le poids et la composition du foie ; les quantités totales de lipides et de protéines hépatiques sont beaucoup plus élevées chez les animaux gavés avec l'aliment TPH (mais + gluten) que chez ceux ayant ingéré l'aliment TPB (mais + amidon). Là encore, on voit que le gavage entraîne non seulement une accumulation de lipides mais de protéines.

\section{TABLEAU 5}

Infuence du taux protidique de l'aliment sur le poids et la composition du foie chez des poulettes ágées de 10 semaines

(Expérience no 3 : gavage de I I jours)

\begin{tabular}{|c|c|c|c|c|}
\hline Lot & $\begin{array}{l}\text { Poids } \\
\text { du foie } \\
\text { (g) }\end{array}$ & $\begin{array}{l}\text { Matières } \\
\text { sèches } \\
\text { du foie } \\
\text { (g) }\end{array}$ & $\begin{array}{l}\text { Protéines } \\
\text { totales } \\
\text { (g) }\end{array}$ & $\begin{array}{l}\text { Lipides } \\
\text { totaux } \\
\text { (g) }\end{array}$ \\
\hline $\begin{array}{l}\text { TPH (mais }+ \text { gluten) } \\
\text { TPB (mais }+ \text { amidon) } \\
\text { Non gavés } \ldots \ldots \ldots\end{array}$ & $\begin{array}{l}79,5^{\mathbf{c}} \\
59,8^{b} \\
33,4^{a}\end{array}$ & $\begin{array}{l}30,7^{c} \\
21,4^{b} \\
14,0^{a}\end{array}$ & $\begin{array}{l}16,0^{b} \\
12,9^{a} \\
10,8^{a}\end{array}$ & $\begin{array}{r}10,13^{b} \\
5,40^{a} \\
2,10^{a}\end{array}$ \\
\hline
\end{tabular}

Les moyennes accompagnées d'une même lettre ne sont pas siguificativement différentes au seuil 0,05 . 


\section{Expérience $n^{\circ} 4$}

Ces résultats sont rapportés dans le tableau 6 . On y remarque que l'élévation de la teneur énergétique du régime par incorporation d'huile ne permet pas d'obtenir des foies plus lourds; ils sont cependant plus riches en matières sèches et en lipides. $\mathrm{Au}$ contraire, la quantité totale de protéines est plus faible avec le régime riche en matières grasses ; cette différence pouvant s'expliquer en partie par un apport alimentaire un peu plus faible en protéines.

\section{TABLEAU 6}

Influence de la teneur énergétique du régime sur le poids et la composition du foie chez des poulettes de 10 semaines

(Expérience $n^{\circ} 4: 15$ jours de gavage)

\begin{tabular}{|c|c|c|c|c|c|}
\hline Lot & $\begin{array}{l}\text { Poids du foie } \\
\text { (g) }\end{array}$ & $\begin{array}{c}\text { Matières sèches } \\
\text { du foie } \\
\text { (g) }\end{array}$ & $\begin{array}{l}\text { Protéines } \\
\text { totales } \\
\text { (g) }\end{array}$ & $\begin{array}{l}\text { Lipides } \\
\text { totaux } \\
\quad(g)\end{array}$ & $\begin{array}{l}\text { Aliment } \\
\text { ingéré } \\
\text { (g) }\end{array}$ \\
\hline $\begin{array}{l}\text { T (mais) } \ldots \ldots \\
\text { TEH (mais }+ \text { huile) } \\
\text { Non gaves } \ldots \ldots \ldots\end{array}$ & $\begin{array}{l}83,5^{b} \\
75,6^{b} \\
51,4^{a}\end{array}$ & $\begin{array}{l}27,0^{b} \\
28,2^{b} \\
14,2^{a}\end{array}$ & $\begin{array}{l}14,7^{b} \\
13,3^{b} \\
11,0^{a}\end{array}$ & $\begin{array}{r}7,52^{a b} \\
11,96^{a} \\
2,34^{b}\end{array}$ & $\begin{array}{ll}3 & 091 \\
3 & 136\end{array}$ \\
\hline
\end{tabular}

Les moyennes accompagnées d'une même lettre ne sont pas significativement différentes.

\section{Expérience no 5}

Les effets de la supplémentation en lysine seule ou en lysine et choline font l'objet du tableau 7. Les différences entre lots gavés ne sont pas significatives.

\section{TABLEAU 7}

Influence de la lysine et de la choline sur le poids et la composition du foie chez des poulettes de 10 semaines

(Expérience $n^{0} 5: 15$ jours de gavage)

\begin{tabular}{|c|c|c|c|c|}
\hline Lot & $\begin{array}{l}\text { Poids } \\
\text { du foie } \\
\text { (g) }\end{array}$ & $\begin{array}{l}\text { Matières } \\
\text { sèches } \\
\text { du foie } \\
\text { (g) }\end{array}$ & $\begin{array}{l}\text { Lipides } \\
\text { totaux } \\
\text { (g) }\end{array}$ & $\begin{array}{c}\text { Aliment } \\
\text { ingéré }\end{array}$ \\
\hline TPH (maïs + gluten) .... & $55,4^{b}$ & $20,8^{b}$ & $4,7^{b}$ & 2664 \\
\hline $\begin{array}{l}\text { VBC (mais }+ \text { gluten }+ \\
\text { choline }+ \text { lysine) } \ldots \ldots \\
\text { VB (mais }+ \text { gluten }+ \text { lysine) } \\
\text { Non gavés } \ldots \ldots \ldots \ldots \ldots\end{array}$ & $\begin{array}{l}60,4^{b} \\
49,7^{b} \\
35,4^{a}\end{array}$ & $\begin{array}{l}24,1^{b} \\
18,8^{b a} \\
11,4^{a}\end{array}$ & $\begin{array}{r}11,7^{b} \\
7,6^{b} \\
1,9^{a}\end{array}$ & $\begin{array}{l}2870 \\
2687\end{array}$ \\
\hline
\end{tabular}


On peut toutefois constater que l'amélioration de la valeur biologique par la lysine ne modifie guère le poids et la composition du foie. L'addition de lysine et de choline permet d'obtenir des foies plus gros et un peu plus gras.

\section{Expérience $n^{\circ} 6$}

Les résultats de cette expérience figurent dans le tableau 8. Ils permettent de comparer un régime complet et équilibré à un régime très déficient composé de maîs. Les différences entre lots gavés ne sont pas significatives. On note une tendance des foies du lot recevant l'aliment complet à être plus petits et moins gras. Cependant, les protéines totales s'accroissent dans les mêmes proportions sous l'effet du gavage.

\section{TABLEAU 8}

Comparaison des effets du mais et d'un régime complet sur le poids et la composition du foie chez des poulettes âgées de 10 semaines

(Expérience no $6:$ I5 jours de gavage)

\begin{tabular}{|c|c|c|c|c|c|}
\hline Lot & $\begin{array}{l}\text { Poids du foie } \\
\text { (g) }\end{array}$ & $\begin{array}{c}\text { Matières sèches } \\
\text { du foie } \\
\text { (g) }\end{array}$ & $\begin{array}{l}\text { Protéines } \\
\text { totales } \\
(\mathrm{g})\end{array}$ & $\begin{array}{l}\text { Lipides } \\
\text { totaux } \\
\text { (g) }\end{array}$ & $\begin{array}{l}\text { Aliment } \\
\text { ingéré } \\
\text { (g) }\end{array}$ \\
\hline $\begin{array}{l}\text { T (maïs) } \ldots \ldots \ldots \ldots \ldots \\
\mathrm{C} \text { (régime complet) } \ldots \ldots \\
\text { Non gavés } \ldots \ldots \ldots \ldots \ldots\end{array}$ & $\begin{array}{l}63,2^{b} \\
55,6^{b} \\
44,1^{b}\end{array}$ & $\begin{array}{l}20,9^{b} \\
17,9^{b} \\
12,6^{a}\end{array}$ & $\begin{array}{l}12,10^{b} \\
12,45^{b} \\
10,10^{a}\end{array}$ & $\begin{array}{l}5,54 b \\
4,05 b \\
1,77^{a}\end{array}$ & $\begin{array}{l}2366 \\
2360\end{array}$ \\
\hline
\end{tabular}

Les moyennes accompagnées de la même lettre ne sont pas significativement différentes au seuil 0,05 .

\section{DISCUSSION}

Chez les Mammifères, les régimes renfermant peu de protéines ou des protéines déséquilibrées favorisent l'apparition du foie gras (LuCAs et RIDouT, I967). I1 en est de même des régimes carencés en choline (LuCAs et Ridout, I967; Orson, I97I). Nos résultats, comme ceux de BuUM et al., x97I, montrent qu'il en est tout autrement de la stéatose des oiseaux provoquée par une suralimentation. En effet, non seulement l'élévation du taux protidique ne réduit pas le poids du foie et sa teneur en lipides, mais encore, dans plusieurs expériences, elle exagère l'hépatomégalie et la stéatose. Il en est de même de la supplémentation en lysine et de la supplémentation en choline.

Plusieurs mécanismes peuvent être à l'origine de la stéatose. Elle peut provenir soit d'une rétention des lipides au niveau du foie sans changement de l'intensité de leur synthèse, soit d'une augmentation de la lipogenèse sans modification de la quantité de lipides transportés du foie vers la périphérie, soit enfin d'une lipogenèse accrue accompagnée d'une rétention des lipides dans le foie. Le foie gras par suralimentation semble répondre au deuxième schéma. En effet, l'augmentation de la lipogenèse res- 
sort de l'évolution de la composition chimique du foie au cours du gavage de l'Oie (LECLERCQ et al., I968). Il doit en être de même chez le Poulet puisque, comme chez l'Oie, on assiste à un accroissement notable des protéines hépatiques sous l'effet de la suralimentation forcée. Il y aurait donc un accroissement important de l'anabolisme hépatique se traduisant en particulier par une lipogenèse intense. De plus, le transfert des triglycérides du foie vers le sang ne doit pas être très modifié puisque ni la choline, ni les protéines alimentaires ne diminuent la stéatose. Bien au contraire, ces dernières semblent favoriser l'hypertrophie cellulaire et le développement $\mathrm{d} u$ foie gras. Certes, l'absence d'effet bénéfique du régime complet vient nuancer cette opinion. Des facteurs nutritionnels (minéraux, vitamines ou acides aminés autres que la lysine) peuvent sans doute empêcher la stéatose en permettant la mobilisation des graisses vers la périphérie.

En définitive, un apport correct de nutriments essentiels à l'hépatocyte et en premier lieu d'acides aminés semble indispensable pour permettre au foie d'une part de manifester une activité de synthèse élevée, d'autre part de conserver une structure cellulaire compatible avec la surcharge lipidique. Cette hypothèse sera l'objet de nos prochaines investigations.

Reçu pour publication en juillet 1971.

\title{
SUMMARY
}

\section{DIETARY FACTORS ENHANCING STEATOSIS}

IN THE FORCE-FED CHICKEN

\begin{abstract}
The effects of various dietary components were investigated on chickens force-fed by a cramming-machine. The weights, the lipid and protein rates of livers increased depending on dietary protein rate. Peanut oil supplementation induced an increase in energy nutriment intake; it increased steatosis, and had no effect on liver weight. Steatosis and hepatomegalia were not decreased by improving dietary value with lysine or by adding choline to the diet. There was no significant difference between chickens force-fed corn vs. whole diet. Therefore, steatosis in the force-fed chicken appears to be due not to dietary deficiency in protein or lipotropic factors, but to an increased lipid synthesis. Appropriate protein rate seems necessary to allow high anabolic activity of the liver and cellular integrity maintenance.
\end{abstract}

\section{RÉFÉRENCES BIBLIOGRAPHIQUES}

Blum J.-C., Monachon G., Leclerce B., I97I. Liver steatosis of force fed geese as influenced by the protein level of the diet. Act. Vet. Acad. Sci. Hung., (sous presse).

Leclerce B., Durand G., Delpech P., Blum J.-C., I968. Note préliminaire sur l'évolution des constituants biochimiques du foie au cours du gavage de l'oie. Ann. Biol. anim. Bioch. Biophys., 8, 549-556.

Lucas C. C., Ridout J. H., 1967. Progress in the Chemistry of fats and others lipids. Part. 1 : Fatty livers and lipotropic phenomena. Pergamon Press.

OLSON R. E., I97I. Scientific contribution of Wendell Griffith to our understanding of the function of choline. Fed. Proc., 30, 13r-1 38 . 